\title{
Two-Year Multicenter Outcomes of iStent inject Trabecular Micro-Bypass Stents Combined with Phacoemulsification in Various Types of Glaucoma and Ocular Hypertension
}

This article was published in the following Dove Press journal:

Clinical Ophthalmology

\author{
Colin Clement $\mathbb{D}^{1-4}$ \\ Frank Howes (iD ${ }^{5}$ \\ Alexandros S loannidis (iD) \\ Michael Shiu ${ }^{7}$ \\ David Manning (1D ${ }^{8}$ \\ Jed Lusthaus $\mathbb{1}^{3,4,9}$ \\ Ridia Lim $\mathbb{1 D}^{3,10}$ \\ Simon E Skalicky (D) ${ }^{11}$ \\ Todd Goodwin ${ }^{12}$
}

'Eye Associates, Sydney, NSW, Australia; ${ }^{2}$ Fairfield Eye Surgery, Fairfield, NSW, Australia; ${ }^{3}$ Glaucoma Unit, Sydney Eye Hospital, Sydney, NSW, Australia; ${ }^{4}$ Discipline of Ophthalmology, The University of Sydney, Sydney, NSW, Australia; ${ }^{5}$ Eye \& Laser Centre, Gold Coast, QLD, Australia; ${ }^{6}$ Vision Eye Institute, Melbourne, VIC, Australia; ${ }^{7}$ Laser Sight Centre, Melbourne, VIC, Australia; ${ }^{8}$ Hunter Cataract \& Eye Centre, Charlestown, NSW, Australia; ${ }^{9}$ Eyehaus, Sydney, NSW, Australia; ${ }^{10}$ Hunter St. Eye Specialists, Parramatta, NSW, Australia; "'Department of Surgery Ophthalmology, University of Melbourne, Melbourne, VIC, Australia; ${ }^{2} \mathrm{NQ}$ Eye Specialists, Currajong, QLD, Australia
Correspondence: Colin Clement Eye Associates, Level 4, 187 Macquarie Street, Sydney, NSW 2000, Australia Tel +6I 292479972

Email colinandkylie@me.com
Purpose: This multicenter study evaluated 2-year effectiveness and safety following implantation of two second-generation trabecular micro-bypass stents (iStent inject $^{\circledR}$ ) with phacoemulsification. Materials and Methods: This was a retrospective study of iStent inject implantation with phacoemulsification by nine surgeons across Australia. Eyes had mild to advanced glaucoma (predominantly primary open-angle/POAG, appositional angle-closure/ACG, or normal-tension /NTG) or ocular hypertension (OHT), and cataract. Evaluations included intraocular pressure (IOP); medications; proportions of eyes with 0 or $\geq 2$ medications, reduced/stable medications versus preoperative, and IOP $\leq 15 \mathrm{mmHg}$; visual acuity; cup-to-disc ratio (CDR); visual fields (VF); adverse events; and secondary surgery.

Results: A total of 340 eyes underwent surgery and had 24-month follow-up data. At 24 months, mean IOP decreased by $16 \%$ from $16.4 \pm 4.7 \mathrm{mmHg}$ preoperatively to $13.7 \pm 3.1 \mathrm{mmHg}(\mathrm{p}<0.001)$, and $77 \%$ of eyes achieved IOP of $\leq 15 \mathrm{mmHg}$ versus $49 \%$ preoperatively ( $<<0.001$ ). Mean number of medications decreased by $67 \%$ to $0.49 \pm 0.95$ versus $1.49 \pm 1.20$ preoperatively $(\mathrm{p}<0.001$ ), with $74 \%$ of eyes medication-free versus $25 \%$ preoperatively $(\mathrm{p}<0.001$ ), and $14 \%$ of eyes on $\geq 2$ medications versus $46 \%$ preoperatively $(\mathrm{p}<0.001$ ). Medication burden was reduced or stable in $98 \%$ of eyes versus preoperative. Stratified analyses showed significant IOP and medication reductions across glaucoma subtypes (POAG, ACG, NTG, OHT): 13-22\% for IOP ( $<<0.01$ for all) and $62-100 \%$ for medication ( $\mathrm{p}<0.001$ for all). Favorable safety included few adverse events; stable CDR, VF, and visual acuity; and filtering surgery in only 8 eyes $(2.4 \%)$ over 2 years.

Conclusion: This 340-eye multicenter dataset provides robust evidence of the safety and efficacy of iStent inject implantation with phacoemulsification, with significant and sustained IOP and medication reductions through 2 years. Results were similarly favorable across glaucoma subtypes (including POAG, ACG, NTG, OHT) and were attained across various glaucoma severities, clinical sites, and surgeons, highlighting the real-world versatility and utility of this treatment modality.

Keywords: microinvasive glaucoma surgery, MIGS, glaucoma, iStent inject, intraocular pressure, second-generation, multicenter

\section{Introduction}

As the leading cause of irreversible blindness worldwide, glaucoma is expected to increase in prevalence even further in coming years, expanding from 76 million patients affected in 2020 to 95.4 million patients in $2030 .{ }^{1}$ Glaucoma treatment focuses on decreasing intraocular pressure (IOP), which is typically achieved 
through ocular hypotensive medication, laser therapy, micro-invasive glaucoma surgery (MIGS), and/or traditional filtering surgeries such as trabeculectomy or tube shunt. $^{2}$

For a given patient, the choice of treatment often depends on numerous factors, such as disease severity, rate of progression, treatment compliance, medication hypersensitivities, or surgical and medical history. Along the spectrum of treatment options, MIGS procedures have increasingly filled a critical gap that previously existed between more conservative therapies (such as medications) and higher-risk filtering surgeries. The IOP reduction achieved with MIGS devices is expected to be more modest than that of filtering surgery, and MIGS procedures are typically employed earlier in the glaucoma treatment algorithm than filtering surgery. However, in an overall risk-benefit calculation for a given patient, it is important to consider the risks of filtering surgery (eg, endophthalmitis, hypotony, blebitis, choroidal hemorrhage or effusion $)^{3-5}$ as well as its five-year failure rates $(\sim 50 \%){ }^{3}$

The iStent $^{\circledR}$ trabecular micro-bypass stent (Glaukos Corporation, San Clemente, CA, USA) was the first MIGS device approved by the US Food and Drug Administration (FDA). It has been the subject of some of the most broad-ranging research to-date of the MIGS devices, with outcomes through seven years in a variety of glaucoma subtypes, and in both standalone usage or combined with cataract surgery. ${ }^{6-22}$ The more recently introduced iStent inject trabecular micro-bypass, containing two stents with second-generation design, has quickly gained traction as well, with outcomes reported through four years postoperative. ${ }^{23-39}$ The evidence for both devices has consistently shown sustained IOP and medication reductions alongside a favorable safety profile.

A prior publication ${ }^{23}$ reported 1-year multicenter outcomes of iStent inject implantation with cataract surgery in 165 eyes from five surgeons in Australia. The cohort included various subtypes of glaucoma, with the most predominant diagnoses being primary open-angle glaucoma (POAG), appositional angle-closure glaucoma (ACG), pseudoexfoliative glaucoma (PXG), and ocular hypertension (OHT). The results showed a 23.2\% IOP reduction and $71.5 \%$ medication reduction from preoperative, with over three-fourths of patients medication-free (on zero medications) at 12 months (vs 17.6\% preoperatively). The present report shows 2-year outcomes from these five surgeons as well as from four additional surgeons, for a total of 340 eyes from nine surgeons across Australia.

\section{Materials and Methods Study Population}

This study was a retrospective outcome assessment of pooled data from nine surgeons across Australia. Records were reviewed to identify eyes implanted with iStent inject in the setting of cataract surgery between January 2016 and February 2018. Allowed diagnoses included primary open-angle glaucoma, pseudoexfoliative glaucoma, normal-tension glaucoma, pigmentary glaucoma, combinedmechanism glaucoma, appositional angle-closure glaucoma (ACG) and ACG suspects, or ocular hypertension. Consistent with clinical and regulatory guidelines, all eyes were eligible for cataract surgery and needed additional glaucoma intervention due to inadequate IOP control, visual field progression, heavy medication burden, and/or nonadherence with topical therapy; eyes were not eligible for iStent inject surgery if they had significant ocular comorbidities, active ocular inflammation, or synechial angle closure. Efficacy was quantified by mean IOP and number of medications; and proportional analyses of eyes on zero medications, eyes on $\geq 2$ medications, eyes with the same or decreased medication burden versus preoperative, and eyes with IOP $\leq 15 \mathrm{mmHg}$. Safety outcomes included corrected distance visual acuity (CDVA), cup-to-disc ratio (CDR), visual field (VF), surgical complications, postoperative adverse events, and secondary surgical interventions.

\section{iStent inject Device and Implantation Technique}

The iStent inject trabecular micro-bypass system contains two biocompatible heparin-coated titanium stents, each with four lateral outlet lumens to allow for multidirectional aqueous egress from the anterior chamber. The stents are pre-loaded in a single-use sterile injector that is advanced ab internally under gonioscopic guidance through the phacoemulsification incision at the close of cataract surgery. After advancing the injector to the nasal angle, the stents are implanted two clock hours apart through two separate regions of the trabecular meshwork into Schlemm's canal. This placement, completed in a single intraocular entry, allows access to up to six clock hours of collector channels for aqueous outflow. ${ }^{39}$ The viscoelastic is then removed, the eye is irrigated with balanced salt solution, and the 
wound is confirmed to be patent. Following surgery, patients were prescribed each surgeon's standard postoperative medication regimen, which usually included a topical antibiotic for 1-2 weeks and a topical steroid (typically a prednisolone formulation such as prednefrin forte) tapered over 4 weeks.

All patients signed an informed consent to allow for the retrospective evaluation of their de-identified clinical data. All data were collected in accordance with the tenets of the Declaration of Helsinki, and the study received ethics committee approval from the Royal Australian and New Zealand College of Ophthalmology Human Research Ethics Committee. Descriptive statistics were used to summarize pre- and postoperative data, including means $( \pm$ standard deviation) and proportional analyses. Paired t-tests were used to compare pre- and postoperative mean IOP and medication values. The McNemar test was used to compare the proportions of eyes with IOP $\leq 15 \mathrm{mmHg}$ or $\leq 18 \mathrm{mmHg}$, and the proportions of eyes on 0 medications or on $\geq 2$ medications. Results were considered significant for $\mathrm{p}$-values $<0.05$. Patients have been followed for 24 months, and follow-up continues.

\section{Results}

\section{Patient Population}

Of 421 total eyes that underwent cataract surgery with iStent inject trabecular micro-bypass stent implantation, 340 eyes of 230 patients reached 24 months of follow-up by the time of data collection; these eyes constitute the consistent cohort analyzed in this report. Preoperative demographic and ocular characteristics are summarized in Table 1. Most patients were Caucasian, and the most common diagnoses were POAG (67\%), appositional ACG or ACG suspects (10\%), ocular hypertension or glaucoma suspects (8\%), and normal-tension glaucoma (NTG, 6\%). Approximately $35 \%$ of eyes (119/340) had a history of prior glaucoma procedure(s), most commonly a laser trabeculoplasty and/or laser peripheral iridotomy; 2 eyes had undergone prior trabeculectomy, which was nonfunctioning at the time of iStent inject surgery. Approximately $76 \%$ of eyes had mild glaucoma based on visual field criteria (Hodapp-Anderson-Parrish grading scale). ${ }^{40}$

\section{IOP and Medications}

Figures 1 and 2 depict the mean and proportional analysis of IOP from preoperative to 24 months postoperative. At
24 months, mean IOP decreased by $16 \%$ from $16.4 \pm 4.7$ mmHg preoperatively to $13.7 \pm 3.1 \mathrm{mmHg}$ ( $\mathrm{p}<0.001$ ); $77 \%$ of eyes achieved IOP $\leq 15 \mathrm{mmHg}$ versus $49 \%$ preoperatively ( $<<0.001$ ); and $93 \%$ of eyes had IOP $\leq 18 \mathrm{mmHg}$ versus $77 \%$ preoperatively $(\mathrm{p}<0.001)$. Figures 3 and 4 display the mean and proportional analysis of medication burden from preoperative to 24 months postoperative. At 24 months, the mean number of medications decreased by $67 \%$ to $0.49 \pm 0.95$ versus $1.49 \pm 1.20$ preoperatively ( $p<0.001$ ), with $74 \%$ of eyes medication-free versus $25 \%$ preoperatively $(p<0.001)$, and only $14 \%$ of eyes on $\geq 2$ medications versus $46 \%$ preoperatively $(p<0.001)$. Patients' medication regimen decreased or remained the same in all but $98 \%$ of eyes (332/340) versus preoperative.

Outcomes were stratified by glaucoma subtype for the four most prevalent diagnoses in the cohort (POAG $n=227$, ACG $n=35$, OHT $n=28$, NTG $n=20$ ). Within these analyses, significant reductions in IOP and medications were achieved across all glaucoma subtypes. At 24 months versus baseline, mean IOP reduced by $14 \%$ in POAG eyes $(\mathrm{p}<0.001), 19 \%$ in ACG eyes $(\mathrm{p}<0.001)$, $22 \%$ in OHT eyes $(\mathrm{p}<0.001)$, and $13 \%$ in NTG eyes $(\mathrm{p}=0.007)$ [Figure 5]. At 24 months, medication reductions versus baseline were $65 \%$ in POAG eyes, $74 \%$ in ACG eyes, $100 \%$ in OHT eyes, and $62 \%$ in NTG eyes $(p<0.001$ for all) [Figure 6].

\section{Safety}

Successful implantation of two stents was achieved in all but 2 eyes (338/340 or 99.4\%); in those 2 eyes, only 1 stent was implanted but meaningful reductions in IOP and medication were still observed through two years (one eye had final IOP $13 \mathrm{mmHg}$ on 1 medication vs baseline IOP $18 \mathrm{mmHg}$ on 2 medications; the other eye had final IOP 20 mmHg on 1 medication vs baseline IOP $26 \mathrm{mmHg}$ on 3 medications). Over two years of follow-up, there were no adverse events occurring at a rate of $2 \%$ or greater, a threshold frequently used in MIGS clinical studies. ${ }^{24,41}$ The adverse events that did occur were mild, transient, and resolved with minimal to no intervention and without incurring sequelae. Nearly all eyes (96\%) achieved CDVA of $20 / 40$ or better at two years, up from $90 \%$ preoperatively. Mean CDR remained stable at 24 months $(0.71 \pm 0.16)$ versus baseline $(0.69 \pm 0.17)(\mathrm{p}=0.263)$. The average visual field mean deviation also was stable at 24 months $(-4.90 \pm 5.56 \mathrm{~dB})$ versus preoperative $(-4.60 \pm$ $4.93 \mathrm{~dB})(\mathrm{p}=0.191)$ [Table 2]. 
Table I Demographic and Preoperative Ocular Characteristics

\begin{tabular}{|c|c|c|c|c|}
\hline \multirow{3}{*}{ Age (years) (data from 339 eyes) } & \multirow[b]{2}{*}{ Mean \pm SD } & \multicolumn{3}{|c|}{$\begin{array}{l}\mathrm{N}=340 \text { Eyes of } 230 \\
\text { Patients }\end{array}$} \\
\hline & & 73.0 & \pm & 7.7 \\
\hline & Range & 42 & - & 90 \\
\hline Gender (data from 340 eyes) & Male/Female & \multicolumn{3}{|l|}{$43 \% / 57 \%$} \\
\hline \multirow[t]{4}{*}{ Race/Ethnicity (data from 308 eyes) } & White & \multicolumn{3}{|l|}{$95.5 \%$} \\
\hline & Asian & \multicolumn{3}{|l|}{$3.2 \%$} \\
\hline & Hispanic & \multicolumn{3}{|l|}{$0.6 \%$} \\
\hline & Arabic & \multicolumn{3}{|l|}{$0.6 \%$} \\
\hline \multirow[t]{11}{*}{ Type of Glaucoma (data from 340 eyes) } & & \multicolumn{3}{|l|}{$\mathrm{n} \%$} \\
\hline & POAG & \multicolumn{2}{|l|}{227} & $66.76 \%$ \\
\hline & Appositional ACG or ACG suspects & \multicolumn{2}{|l|}{35} & $10.29 \%$ \\
\hline & OHT/glaucoma suspect & \multicolumn{2}{|l|}{28} & $8.24 \%$ \\
\hline & NTG & \multicolumn{2}{|l|}{20} & $5.88 \%$ \\
\hline & PXG & \multicolumn{2}{|l|}{10} & $2.94 \%$ \\
\hline & CMG & \multicolumn{2}{|l|}{5} & $1.47 \%$ \\
\hline & PG & \multicolumn{2}{|l|}{10} & $2.94 \%$ \\
\hline & Uveitic & \multicolumn{2}{|l|}{3} & $0.88 \%$ \\
\hline & Neovascular & \multicolumn{2}{|l|}{ I } & $0.29 \%$ \\
\hline & Angle recession & \multicolumn{2}{|l|}{1} & $0.29 \%$ \\
\hline \multirow[t]{2}{*}{ Eyes with prior glaucoma surgical or laser procedures (data from 340 eyes) } & No & \multicolumn{2}{|l|}{221} & $65.0 \%$ \\
\hline & $Y_{e s}{ }^{\mathrm{a}}$ & \multicolumn{2}{|l|}{119} & $35.0 \%$ \\
\hline$C: D$ ratio (data from 310 eyes) & Mean \pm SD & 0.70 & \pm & 0.17 \\
\hline CDVA $20 / 40$ or better (data from 340 eyes) & $\%(n / N)$ & \multicolumn{3}{|c|}{$89.7 \%(305 / 340)$} \\
\hline VF MD (dB) (data from 299 eyes) & Mean \pm SD & -4.619 & \pm & 4.998 \\
\hline Central Corneal Thickness $(\mu \mathrm{m})$ (data from 267 eyes) & Mean \pm SD & 537 & \pm & 37 \\
\hline \multirow[t]{3}{*}{ Glaucoma Severity ${ }^{\mathrm{b}}$} & Mild & 227 & & $75.9 \%$ \\
\hline & Moderate & 50 & & $16.7 \%$ \\
\hline & Severe & 22 & & $7.4 \%$ \\
\hline IOP (mmHg) (data from 340 eyes) & Mean \pm SD & 16.4 & \pm & 4.7 \\
\hline \multirow[t]{2}{*}{ Number of medications (data from 340 eyes) } & Mean \pm SD & 1.49 & \pm & 1.20 \\
\hline & Range & 0 & - & 5 \\
\hline
\end{tabular}

Notes: Not all preoperative measurements were available for all eyes. ${ }^{a}$ Includes 117 eyes with prior laser procedures [laser peripheral iridotomy and/or laser trabeculoplasty] and 2 eyes with prior trabeculectomy that was no longer functioning; some eyes had more than one prior procedure. ${ }^{\circ}$ Consistent with HodappAnderson-Parrish visual field criteria. Mild: VF MD no worse than $-6 \mathrm{~dB}$, moderate: VF MD worse than $-6 \mathrm{~dB}$ but no worse than $-12 \mathrm{~dB}$, severe: VF MD worse than $-12 \mathrm{~dB}$. Abbreviations: POAG, primary open-angle glaucoma; ACG, appositional angle-closure glaucoma; OHT, ocular hypertension; NTG, normal-tension glaucoma; PXG, pseudoexfoliative glaucoma; CMG, combined-mechanism glaucoma; PG, pigmentary glaucoma; IOP, intraocular pressure; SD, standard deviation; CDVA, corrected distance visual acuity; MD, mean deviation; VF, visual field; C:D, cup-to-disc. 


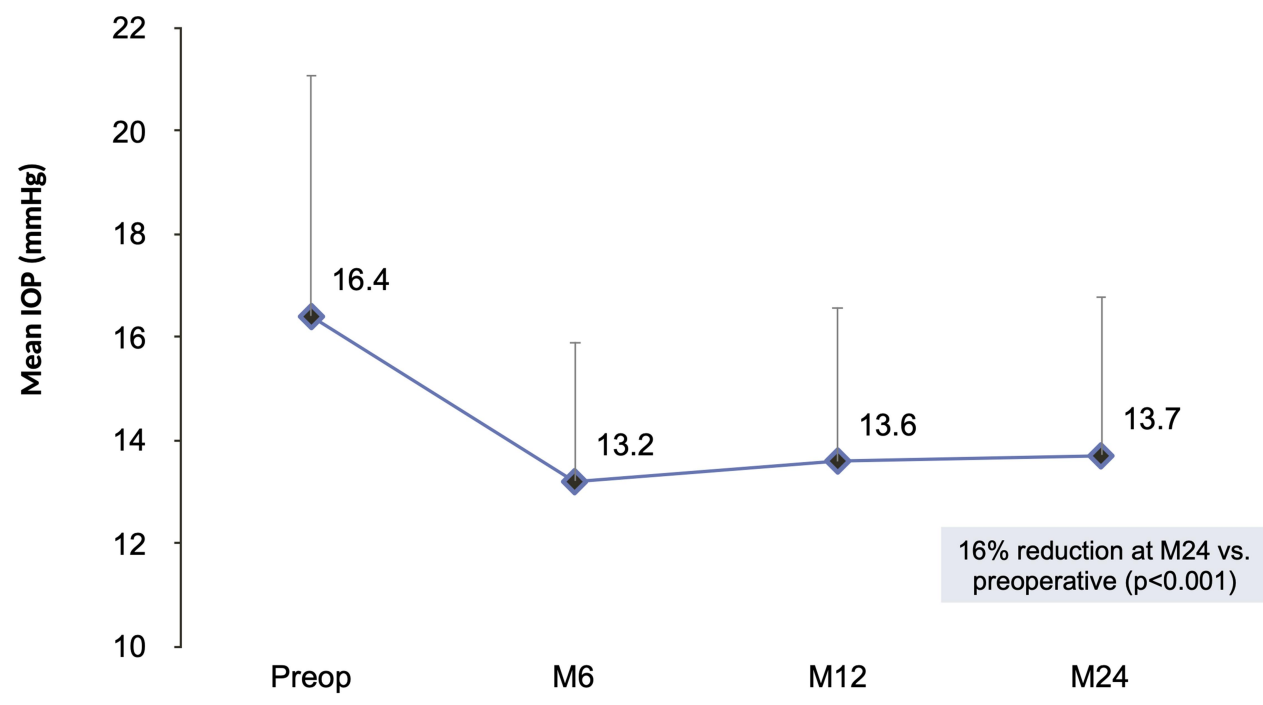

Figure I Mean IOP through 24 months postoperative, all eyes $(n=340)$.

Abbreviations: IOP, intraocular pressure; M, month; Preop, preoperative.

100

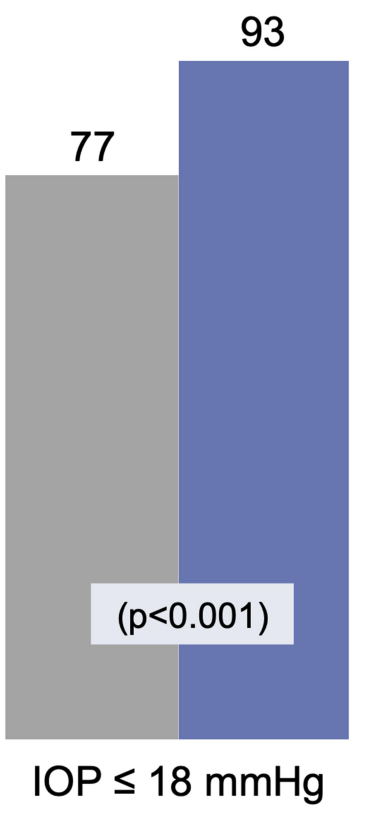

\section{$\mathrm{IOP} \leq 15 \mathrm{mmHg}$}

\section{Preop}

77

75

50

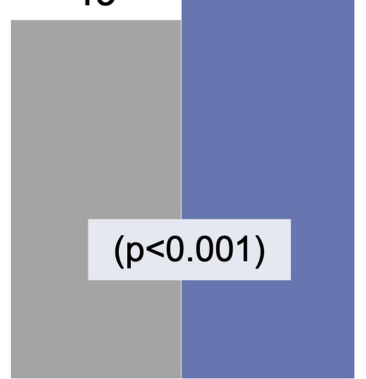

Figure 2 Proportional analysis of IOP at 24 months vs preoperative, all eyes $(n=340)$. Abbreviations: IOP, intraocular pressure; Preop, preoperative.

Secondary glaucoma procedures were infrequent, with the majority noted to be unrelated to the stent and/or due to disease progression despite good IOP control. Over two years of follow-up, a total of 6 eyes had filtration surgery alone, 5 eyes had selective laser trabeculoplasty (SLT) alone, and 2 eyes had both filtration surgery and SLT (hence 8 total filtration surgeries and 7 total SLT procedures in a total of 13 eyes); in addition, 2 eyes underwent implantation of a second iStent inject. Final IOP at two years in eyes receiving secondary interventions ranged from 5 to $17 \mathrm{mmHg}$ on a range of 0 to 4 medications. In the eyes receiving a second iStent inject specifically, final IOP at two years was $7 \mathrm{mmHg}$ on 0 medications and $14 \mathrm{mmHg}$ on 0 medications, respectively; 


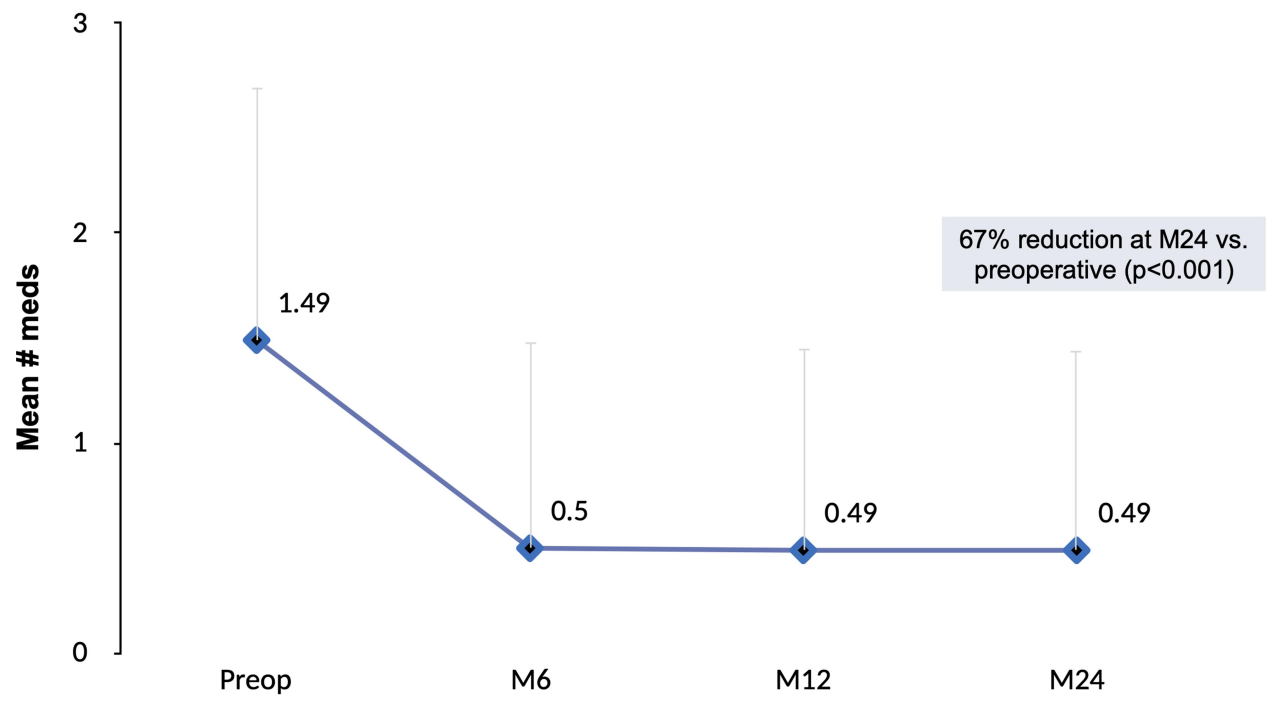

Figure 3 Mean number of medications through 24 months postoperative, all eyes $(n=340)$.

Abbreviations: M, month; Preop, preoperative; Meds, medications.

100
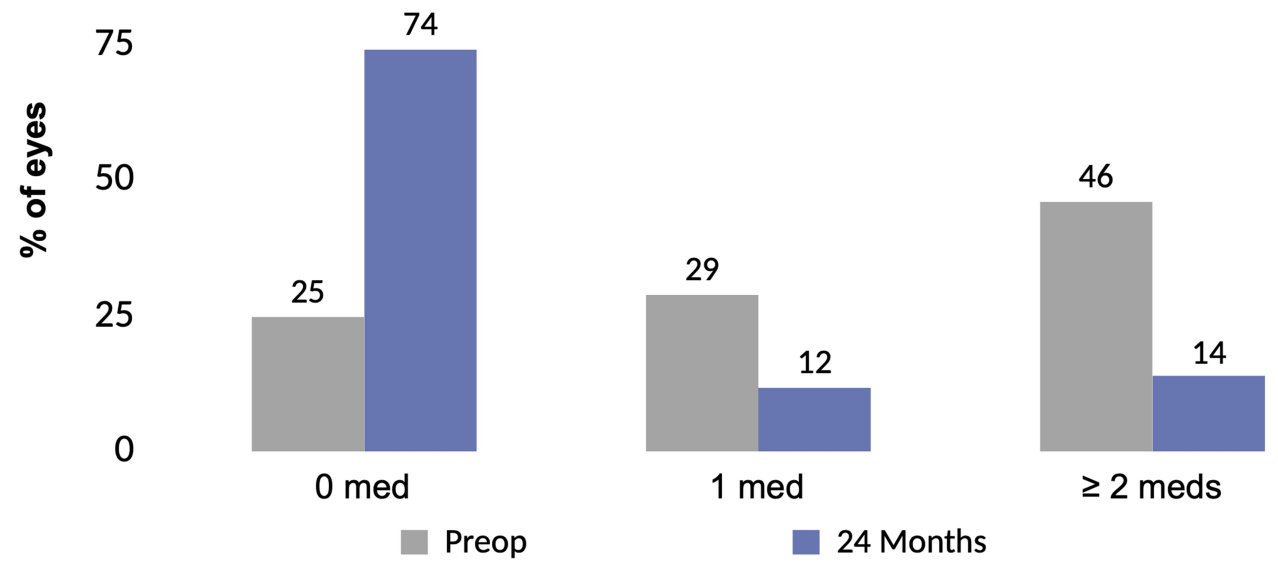

Figure 4 Proportional analysis of medications at 24 months vs preoperative, all eyes $(n=340)$.

Abbreviations: Preop, preoperative; Meds, medications.

no stent-related adverse events or anomalies were reported for either eye.

\section{Discussion}

This large multicenter real-world case series presents sustained safety and effectiveness outcomes through 2 years postoperative following iStent inject implantation with concomitant cataract surgery. The cohort includes various glaucoma subtypes and severities, as well as data from various surgeons and sites across Australia, thereby increasing its clinical applicability and generalizability to other populations. Within this environment of real-world clinical practices and patient populations, iStent inject implantation with phacoemulsification resulted in significant and sustained reductions in IOP and medications through 2 years postoperative. Results were similarly favorable regardless of glaucoma subtype (including POAG, ACG, OHT, NTG). To our awareness, this report represents one of the largest multicenter multi-surgeon real-world studies of iStent inject to-date in the literature.

The observed $2.7 \mathrm{mmHg}$ IOP reduction should be appreciated in the context of the cohort's relatively low mean starting IOP, given that lower starting IOP usually results in smaller postoperative IOP reductions. ${ }^{16,17,29,42,43}$ 

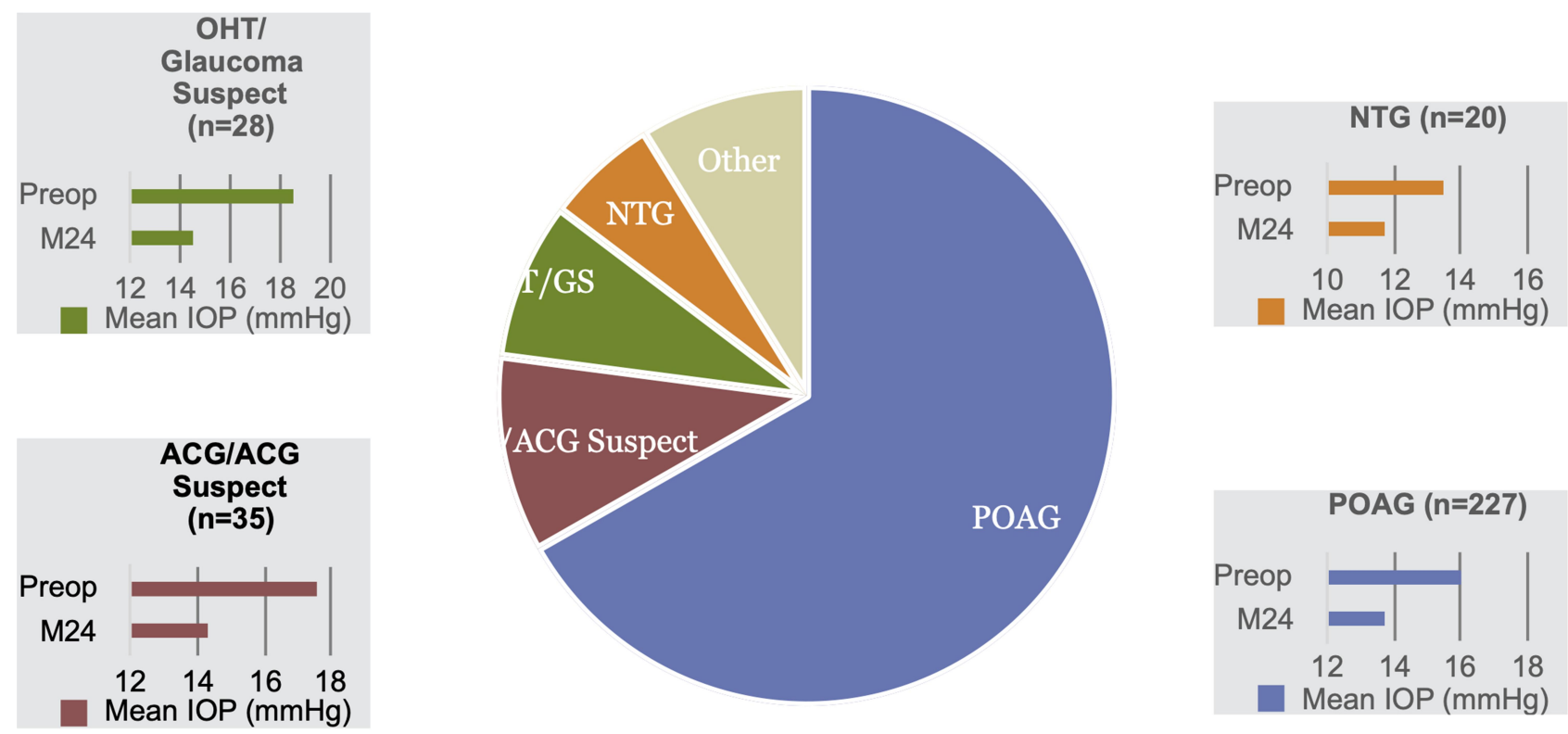

- POAG $\odot$ ACG/ACG Suspect $\odot$ OHT/GS $\odot$ NTG $\odot$ Other

Figure 5 IOP reduction by glaucoma subtype $(p<0.01$ for all).

Abbreviations: IOP, intraocular pressure; POAG, primary open-angle glaucoma; ACG, appositional angle-closure glaucoma; OHT/GS, ocular hypertension/glaucoma suspect; NTG, normal-tension glaucoma; M, month.

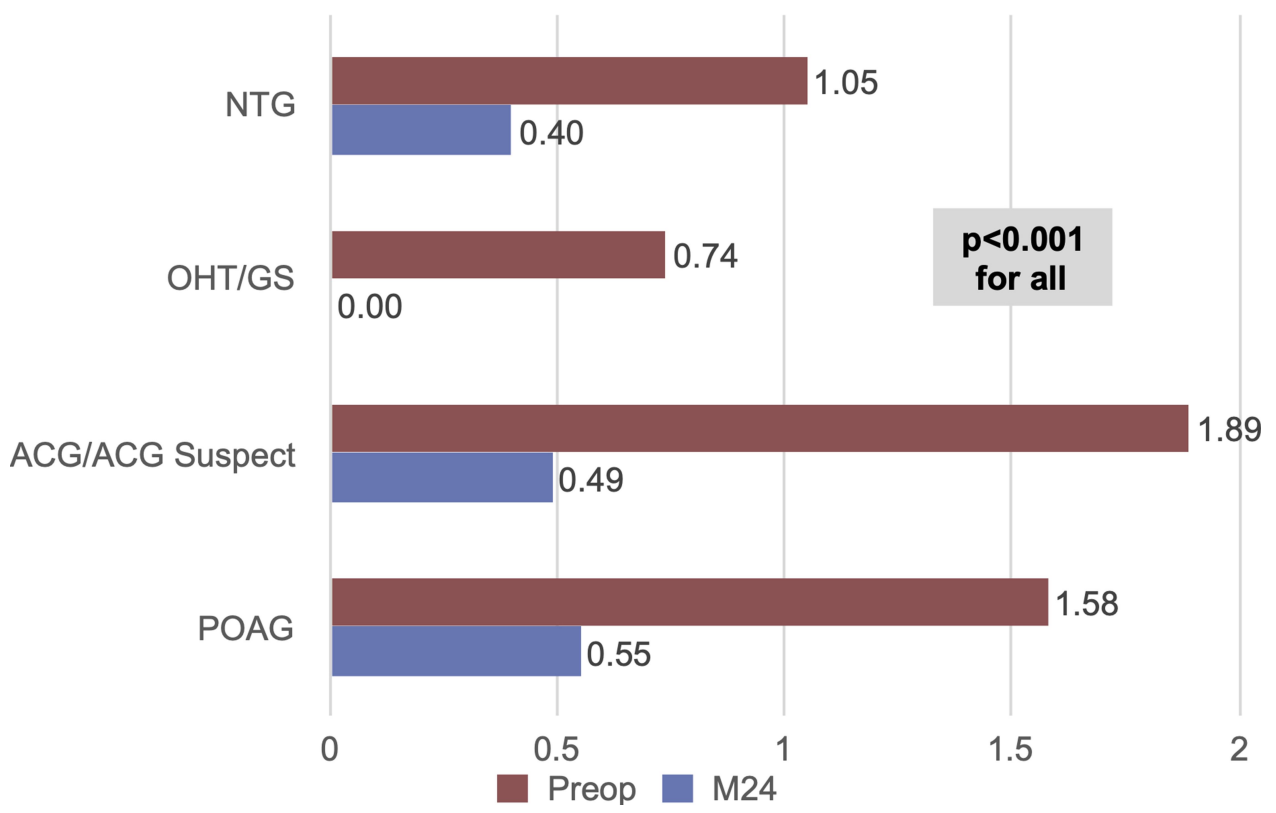

Figure 6 Medication reduction by glaucoma subtype $(\mathrm{p}<0.00 \mathrm{I}$ for all).

Abbreviations: POAG, primary open-angle glaucoma; ACG, appositional angle-closure glaucoma; OHT/GS, ocular hypertension/glaucoma suspect; NTG, normal-tension glaucoma; $\mathrm{M}$, month.

The IOP target of $\leq 15 \mathrm{mmHg}$ was achieved in half-again more eyes at two years versus preoperative ( $77 \%$ vs $49 \%$, $\mathrm{p}<0.001)$. Coinciding with this IOP reduction, there was a threefold decrease in mean medication burden $(0.49$ versus 1.49 medications, $\mathrm{p}<0.001)$. In terms of perpatient medication usage, there was a threefold decline in the proportion of eyes on $\geq 2$ medications versus preoperative $(14 \%$ versus $46 \%, \mathrm{p}<0.001)$, as well as a tripling of 
Table 2 Preoperative and Month 24 CDR, VF MD, and CDVA Consistent Sets of Eyes with Data at Both Time Points

\begin{tabular}{|c|c|c|}
\hline & Preoperative & Year 2 \\
\hline & n (\%) & n (\%) \\
\hline No. of eyes with CDVA recorded at both time points & \multicolumn{2}{|l|}{335} \\
\hline CDVA $20 / 40$ or better & 301 (89.9\%) & $323(96.4 \%)$ \\
\hline No. of eyes with CDR recorded at both time points & \multicolumn{2}{|l|}{213} \\
\hline Mean \pm SD & $0.70 \pm 0.17$ & $0.71 \pm 0.16$ \\
\hline p-value & & 0.263 \\
\hline No. of eyes with VF MD recorded at both time points & \multicolumn{2}{|l|}{226} \\
\hline Mean \pm SD & $-4.60 \pm 4.93$ & $-4.90 \pm 5.56$ \\
\hline$p$-value & & 0.191 \\
\hline
\end{tabular}

Abbreviations: SD, standard deviation; CDR, cup-to-disc ratio; CDVA, corrected distance visual acuity; VF MD, visual field mean deviation.

the proportion of eyes able to come off medications entirely $(74 \%$ versus $25 \%, p<0.001)$. Nearly all eyes were able to lower or maintain their medication regimen versus preoperative.

The IOP and medication reductions observed in this study were in line with what has been reported in the literature, supporting the veracity of the findings. By significantly reducing both IOP and medications, patients can experience a tangible positive shift in their overall disease state. For a given patient, for example, this could take the form of using fewer drops per day, having fewer droprelated side effects, attaining more consistent IOP control at clinic visits, and/or needing fewer subsequent glaucoma procedures. Regardless of a patient's preoperative goal or postoperative benefit, all patients needed cataract surgery, thereby providing a unique opportunity for stent implantation without adding appreciable safety risks above cataract surgery alone.

The medication reduction observed in this study is particularly impressive given that a full quarter of patients in the cohort were on no medications preoperatively. This patient makeup led to a relatively low baseline medication burden, which conceivably could make it more difficult to achieve postoperative reduction. In addition, approximately a quarter of eyes had moderate or severe glaucoma at baseline (VF MD worse than $-6 \mathrm{~dB}$ ); it is possible that these patients were instructed to continue medication in order to maximize IOP reduction regardless of their surgical outcome, due to their more tenuous optic nerve condition.
Reducing a patient's medication burden carries wideranging benefits, given that medications are well-known to worsen treatment adherence, ocular surface disease, and patient quality of life, as well as to incur financial and caregiving ramifications of repeated drop instillation. ${ }^{44-50}$ Treatment adherence is known to decrease dramatically when patients increase from single to multiple eye drops, ${ }^{44}$ placing particular value on the study's finding of a threefold reduction in eyes on 2 or more medications. In addition, the sheer presence of medication can predispose to ocular surface deterioration, ${ }^{46}$ adding further weight to the study's finding of a threefold increase in eyes becoming medication-free.

Alongside this medication reduction, the avoidance of filtration surgeries in nearly all eyes can be highlighted, given the short- and long-term complications and reoperations known to occur with such surgeries. ${ }^{3-5}$ We do acknowledge that this patient population was generally less advanced than patients who would typically be undergoing filtering surgery; however, the rare incidence of such surgery in our cohort ( $2.4 \%$ over two years) is still likely lower than would be expected without stent implantation (projected to be $\sim 3.1-4.6 \%$ per year in the broader glaucoma population). ${ }^{51}$

Stratified analyses were completed for the four most common diagnoses in the cohort (POAG, ACG, OHT, and NTG). Outcomes revealed significant reductions in IOP and medication burden regardless of glaucoma subtype, with IOP reducing $13-22 \%$ and medications reducing $62-100 \%$ (statistically significant for all). The data in the ACG, OHT, and NTG subgroups are especially valuable 
given that these subtypes are less well represented in clinical research. The observed IOP and mediation reductions are consistent with prior work evaluating iStent or iStent inject implantation in these subtypes, ${ }^{11,12,20,25,27}$ and they contribute some of the first long-term analyses on the use of the second-generation iStent inject specifically in these groups.

The reductions in OHT eyes are particularly remarkable, with $100 \%$ of eyes becoming medication-free alongside a $22 \%$ IOP reduction at 2 years postoperative. This possibly reflects the value of restoring trabecular flow while there is still an intact downstream outflow system in an earlier stage of the disease. In such eyes, the data suggest that earlier intervention with a tissue-sparing, minimally-invasive surgical procedure such as iStent inject potentially could alter the trajectory of disease progression before Schlemm's canal and aqueous collector channels are affected by loss of outflow.

The safety profile was highly favorable, as is characteristic of the iStent inject device. Postoperative adverse events were uncommon, mild, and transient; postphacoemulsification improvements in CDVA were maintained over 2 years; and CDR and VF remained stable.

Certain limitations may be discussed in this study. Given that this was a real-world patient population from the surgeons' clinical practices, preoperative medication washout was not completed, as this could pose a risk of IOP elevation in participating eyes. The surgeons managed patients' medications according to their standard practice rather than formal codified guidelines. However, these choices were generally similar across surgeons; also, any differences would be expected to have negligible impact, since the same effectiveness outcomes were evaluated in the same patients and by the same clinicians throughout follow-up, meaning that changes over time still would be apparent. There was no formal control group of phacoemulsification alone, and thus it was not possible to isolate the effects of the stents versus those of cataract surgery. However, other comparative trials of stent-phaco or iStent inject-phaco versus phaco alone $8,11,15,21,22,24$ can be used as a reference point, and the IOP-lowering effect of cataract surgery would be unlikely to persist to the same degree through 2 years postoperative. ${ }^{52}$ Regression to the mean could have been possible, since diurnal IOP measurements and repeated-day evaluations were not employed; however, this would be expected to have minimal to no impact due to the robust sample size.

\section{Conclusion}

This report constitutes one of the largest multicenter datasets to-date of iStent inject with cataract surgery, and it presents outcomes in patients with a variety of glaucoma subtypes and severities, including those that are less frequently addressed in the research. Results were achieved in realworld settings and clinical populations, and thus are readily applicable to practicing clinicians. The outcomes showed that iStent inject plus phacoemulsification can significantly, safely, and sustainably reduce IOP and medication burden through two years postoperative in real-world clinical usage. These gains were similarly favorable across glaucoma subtypes, indicating the viability and adaptability of this treatment modality in managing glaucoma.

\section{Acknowledgments}

No financial support was given for the work in this study. Editorial assistance and publication processing charges were provided by Glaukos Corporation.

\section{Disclosure}

CC reports personal fees from Allergan, Glaukos, Alcon, Merck, Sharp, Dohme. FH reports personal fees from Glaukos. ASI is a consultant for Glaukos. DM reports personal fees from Glaukos, Allergan, and Alcon including being a consultant for Alcon. JL reports research grants from Sydney Eye Hospital Foundation, Glaukos, and Zeiss. RL reports personal fees from Allergan and is a consultant for Glaukos. SS reports honoraria as a consultant from Glaukos. TG reports personal fees from and serves in the advisory board for Glaukos. The authors report no other conflicts of interest in this work.

\section{References}

1. Tham Y-C, Li X, Wong TY, Quigley HA, Aung T, Cheng C-Y. Global prevalence of glaucoma and projections of glaucoma burden through 2040: a systematic review and meta-analysis. Ophthalmology. 2014;121(11):2081-2090. doi:10.1016/j.ophtha.2014.05.013

2. Jonas JB, Aung T, Bourne RR, Bron AM, Ritch R, Panda-Jonas S. Glaucoma. Lancet. 2017;390(10108):2183-2193. doi:10.1016/S01406736(17)31469-1

3. Jampel HD, Musch DC, Gillespie BW, et al. Perioperative complications of trabeculectomy in the collaborative initial glaucoma treatment study (CIGTS). Am J Ophthalmol. 2005;140(1):16-22. doi:10.1016/j. ajo.2005.02.013

4. Gedde SJ, Herndon LW, Brandt JD, Budenz DL, Feuer WJ, Schiffman JC; Tube Versus Trabeculectomy Study Group. Postoperative complications in the Tube Versus Trabeculectomy (TVT) study during five years of follow-up. Am J Ophthalmol. 2012;153:804-814. doi:10.1016/j.ajo.2011.10.024 
5. Rulli E, Biagioli E, Riva I, et al. Efficacy and safety of trabeculectomy vs nonpenetrating surgical procedures: a systematic review and meta-analysis. JAMA Ophthalmol. 2013;131(12):1573-1582. doi:10.1001/jamaophthalmol.2013.5059

6. Al Habash A, Khan O. Outcomes of combined istent trabecular micro-bypass and cataract surgery for the treatment of open-angle glaucoma in a Saudi Population. Clin Ophthalmol. 2020;14:1573-1580. doi:10.2147/OPTH.S249261

7. Samuelson TW, Katz LJ, Wells JM, Duh Y-J, Giamporcaro JE. Randomized evaluation of the trabecular micro-bypass stent with phacoemulsification in patients with glaucoma and cataract. Ophthalmology. 2011;118(3):459-467. doi:10.1016/j. ophtha.2010.07.007

8. Fea AM, Consolandi G, Zola M, et al. Micro-bypass implantation for primary open-angle glaucoma combined with phacoemulsification: 4-year follow-up. J Ophthalmol. 2015;2015:795357. doi:10.1155/ 2015/795357

9. Belovay GW, Naqi A, Chan BJ, Rateb M, Ahmed IIK. Using multiple trabecular micro-bypass stents in cataract patients to treat open-angle glaucoma. $J$ Cataract Refract Surg. 2012;38 (11):1911-1917. doi:10.1016/j.jcrs.2012.07.017

10. Berdahl JP, Dockter Z, Schweitzer J, Ibach M, Ferguson T. Longterm evaluation of a trabecular microbypass stent with cataract surgery in open-angle glaucoma: 7-year results. Presentation at the Virtual Annual Meeting of the American Society of Cataract and Refractive Surgery (ASCRS): May 16; 2020.

11. Chen DZ, Sng CCA, Sangtam T, et al. Phacoemulsification vs phacoemulsification with micro-bypass stent implantation in primary angle closure and primary angle closure glaucoma: a randomized single-masked clinical study. Clin Exp Ophthalmol. 2020. doi: $10.1111 /$ ceo.13721

12. Neuhann TH, Hornbeak DM, Neuhann RT, Giamporcaro JE. Longterm effectiveness and safety of trabecular micro-bypass stent implantation with cataract surgery in patients with glaucoma or ocular hypertension: 5-year outcomes. J Cataract Refract Surg. 2019;45(3):312-320. doi:10.1016/j.jcrs.2018.10.029

13. Gallardo MJ, Supnet RA. Three-year outcomes of combined trabecular micro-bypass and phacoemulsification in a predominantly Hispanic population with primary open-angle glaucoma. Clin Ophthalmol. 2019;13:869-879. doi:10.2147/OPTH.S189071

14. Chang DF, Donnenfeld ED, Katz LJ, et al. Efficacy of two trabecular micro-bypass stents combined with topical travoprost in open-angle glaucoma not controlled on two preoperative medications: 3-year follow-up. Clin Ophthalmol. 2017;11:523-528. doi:10.2147/OPTH. S121041

15. Scott RA, Ferguson TJ, Stephens JD, Berdahl JP. Refractive outcomes after trabecular microbypass stent with cataract extraction in open-angle glaucoma. Clin Ophthalmol. 2019;13:1331-1340. doi:10.2147/OPTH.S206619

16. Ferguson T, Swan R, Ibach M, Schweitzer J, Sudhagoni R, Berdahl JP. Evaluation of a trabecular microbypass stent with cataract extraction in severe primary open-angle glaucoma. $J$ Glaucoma. 2018;27(1):71-76. doi:10.1097/IJG.0000000000000825

17. Ferguson TJ, Swan RJ, Bleeker A, et al. Trabecular microbypass stent implantation in pseudoexfoliative glaucoma: long-term results [published online ahead of print, 2020 May 7]. J Cataract Refract Surg. 2020. doi:10.1097/j.jcrs.0000000000000243

18. Ferguson TJ, Ibach M, Schweitzer J, Karpuk KL, Stephens JD, Berdahl JP. Trabecular microbypass stent implantation with cataract extraction in pigmentary glaucoma. Clin Exp Ophthalmol. 2019. doi: $10.1111 /$ ceo. 13638

19. Ferguson TJ, Ibach M, Schweitzer J, et al. Trabecular microbypass stent implantation in pseudophakic eyes with open-angle glaucoma: long-term results. J Cataract Refract Surg. 2019;45(4):414-420. doi:10.1016/j.jcrs.2018.11.005
20. Fechtner RD, Voskanyan L, Vold SD, et al. Five-year, prospective, randomized, multi-surgeon trial of two trabecular bypass stents versus prostaglandin for newly-diagnosed open-angle glaucoma. Ophthalmol Glaucoma. 2019;2(3):156-166. doi:10.1016/j. ogla.2019.03.004

21. El Wardani M, Bergin C, Achache F, Sharkawi E. Evaluating the trabecular micro-bypass stent combined with phacoemulsification compared to phacoemulsification alone. Klin Monbl Augenheilkd. 2015;232(4):442-445. doi:10.1055/s-0035-1545798

22. Craven ER, Katz LJ, Wells JM, Giamporcaro JE. Cataract surgery with trabecular micro- bypass stent implantation in patients with mild-to-moderate open-angle glaucoma and cataract: two-year follow-up. $J$ Cataract Refract Surg. 2012;38:1339-1345. doi:10.1016/j.jcrs.2012.03.025

23. Clement CI, Howes F, Ioannidis AS, Shiu M, Manning D. One-year outcomes following implantation of second-generation trabecular micro-bypass stents in conjunction with cataract surgery for various types of glaucoma or ocular hypertension: multicenter, multi-surgeon study. Clin Ophthalmol. 2019;13:491-499. doi:10.2147/OPTH.S187272

24. Samuelson TW, Sarkisian SR Jr, Lubeck DM, et al.; for the iStent inject Study Group. Prospective, randomized, controlled pivotal trial of iStent inject trabecular micro-bypass in primary open-angle glaucoma and cataract: two-year results. Ophthalmology. 2019;126 (6):811-821. doi:10.1016/j.ophtha.2019.03.006

25. Neuhann R, Neuhann T. Second-generation trabecular micro-bypass stent implantation: retrospective analysis after 12- and 24-month follow-up. Eye Vis (Lond). 2020;7:1. doi:10.1186/s40662-019-0169-7

26. Salimi A, Lapointe J, Harasymowycz P. One-year outcomes of second-generation trabecular micro-bypass stents (iStent inject) implantation with cataract surgery in different glaucoma subtypes and severities. Ophthalmol Ther. 2019;8(4):563-575. doi:10.1007/ s40123-019-00214-z

27. Manning D. Real-world case series of iStent or iStent inject trabecular micro-bypass stents combined with cataract surgery. Ophthalmol Ther. 2019;8(4):549-561. doi:10.1007/s40123-01900208-x

28. Guedes RAP, Gravina DM, Lake JC, Guedes VMP, Chaoubah A. One-year comparative evaluation of iStent or iStent inject implantation combined with cataract surgery in a single center. Adv Ther. 2019;36(10):2797-2810. doi:10.1007/s12325-019-01067-5

29. Ferguson TJ, Dockter Z, Bleeker A, et al. iStent inject trabecular microbypass stent implantation with cataract extraction in open-angle glaucoma: early clinical experience. Eye Vis (Lond). 2020;7:28. doi:10.1186/s40662-020-00194-3

30. Hengerer FH, Auffarth GU, Riffel C, Conrad-Hengerer I. Prospective, non-randomized, 36-month study of second-generation trabecular micro-bypass stents with phacoemulsification in various types of glaucoma. Ophthalmol Ther. 2018;7(2):405-415. doi:10.1007/s40123-018-0152-8

31. Hengerer FH, Auffarth GU, Riffel C, Conrad-Hengerer I. Secondgeneration trabecular micro-bypass stents as standalone treatment for glaucoma: a 36-Month Prospective Study. Adv Ther. 2019;36 (7):1606-1617. doi:10.1007/s12325-019-00984-9

32. Hengerer FH. Four-Year Real-World Outcomes of iStent Inject ${ }^{\circledR}$ Trabecular Micro-Bypass Stents. E-Poster Discussion at the Annual Meeting of the American Academy of Ophthalmology (AAO). San Francisco, CA; October 13, 2019.

33. Berdahl J, Voskanyan L, Myers JS, Katz LJ, Samuelson TW. iStent inject trabecular micro-bypass stents with topical prostaglandin as standalone treatment for open-angle glaucoma: 4-year outcomes. Clin Exp Ophthalmol. 2020;48(6):767-774. doi:10.2147/OPTH.S189071

34. Fea AM, Belda JI, Rekas M, et al. Prospective unmasked randomized evaluation of the iStent inject versus two ocular hypotensive agents in patients with primary open-angle glaucoma. Clin Ophthalmol. $2014 ; 8: 875-882$. 
35. Klamann MKJ, Gonnermann J, Pahlitzsch M, et al. iStent inject in phakic open angle glaucoma. Graefes Arch Clin Exp Ophthalmol. 2015;253(6):941-947. doi:10.1007/s00417-015-3014-2

36. Lindstrom R, Sarkisian SR, Lewis R, Hovanesian J, Voskanyan L. Fouryear outcomes of two second-generation trabecular micro-bypass stents in patients with open-angle glaucoma on one medication. Clin Ophthalmol. 2020;14:71-80. doi:10.2147/OPTH.S235293

37. Voskanyan L, Garcia-Feijoo J, Belda JI, Fea A, Junemann A, Baudouin C. Prospective, unmasked evaluation of the iStent inject system for open-angle glaucoma: synergy trial. Adv Ther. 2014;31 (2):189-201. doi:10.1007/s12325-014-0095-y

38. Arriola-Villalobos P, Martinez-de-la-Casa JM, Diaz-Valle D, Morales-Fernandez L, Fernandez-Perez C, Garcia-Feijoo J. Glaukos iStent inject $^{\circledR}$ trabecular micro-bypass implantation associated with cataract surgery in patients with coexisting cataract and open-angle glaucoma or ocular hypertension: a Long-Term Study. J Ophthalmol. 2016;2016:1056573. doi:10.1155/2016/1056573

39. Huang AS, Penteado RC, Papoyan V, Voskanyan L, Weinreb RN. Aqueous angiographic outflow improvement after trabecular micro-bypass in glaucoma patients. Ophthalmol Glaucoma. 2019;2:11-21. doi:10.1016/j.ogla.2018.11.010

40. Hodapp E, Parrish RK II, Anderson DR. Clinical Decisions in Glaucoma. St Louis, MO: The CV Mosby Co; 1993.

41. Samuelson TW, Chang DF, Marquis R, et al.; for the HORIZON Investigators. A schlemm canal microstent for intraocular pressure reduction in primary open-angle glaucoma and cataract: the HORIZON Study. Ophthalmology. 2019;126(1):29-37. doi:10.1016/ j.ophtha.2018.05.012

42. Poley BJ, Lindstrom RL, Samuelson TW. Long-term effects of phacoemulsification with intraocular lens implantation in normotensive and ocular hypertensive eyes. J Cataract Refract Surg. 2008;34 (5):735-742. doi:10.1016/j.jcrs.2007.12.045

43. Shingleton BJ, Gamell LS, O’Donoghue MW, Baylus SL, King R. Long-term changes in intraocular pressure after clear corneal phacoemulsification: normal patients versus glaucoma suspect and glaucoma patients. J Cataract Refract Surg. 1999;25(7):885-890.
44. Robin AL, Novack GD, Covert DW, Crockett RS, Marcic TS Adherence in glaucoma: objective measurements of once-daily and adjunctive medication use. Am J Ophthalmol. 2007;144(4):533-540. doi:10.1016/j.ajo.2007.06.012

45. Leung EW, Medeiros FA, Weinreb RN. Prevalence of ocular surface disease in glaucoma patients. J Glaucoma. 2008;17(5):350-355. doi:10.1097/IJG.0b013e31815c5f4f

46. Baudouin C, Liang H, Hamard P, et al. The ocular surface of glaucoma patients treated over the long term expresses inflammatory markers related to both T-helper 1 and T-helper 2 pathways. Ophthalmology. 2008;115(1):109-115. doi:10.1016/j. ophtha.2007.01.036

47. Nordstrom BL, Friedman DS, Mozaffari E, Quigley HA, Walker AM. Persistence and adherence with topical glaucoma therapy. Am J Ophthalmol. 2005;140(4):598-606. doi:10.1016/j.ajo.2005.04.051

48. Tsai JC. A comprehensive perspective on patient adherence to topical glaucoma therapy. Ophthalmology. 2009;116(11 Suppl):S30-S36. doi:10.1016/j.ophtha.2009.06.024

49. Newman-Casey PA, Blachley T, Lee PP, Heisler M, Farris KB, Stein JD. Patterns of glaucoma medication adherence over four years of follow-up. Ophthalmology. 2015;122(10):2010-2021. doi:10.1016/j.ophtha.2015.06.039

50. Friedman DS, Quigley HA, Gelb L, et al. Using pharmacy claims data to study adherence to glaucoma medications: methodology and findings of the Glaucoma Adherence and Persistency Study (GAPS). Invest Ophthalmol Vis Sci. 2007;48(11):5052-5057. doi:10.1167/ iovs.07-0290

51. Szigiato AA, Trope GE, Jin Y, Buys YM. Trends in glaucoma surgical procedures in Ontario: 1992-2012. Can J Ophthalmol. 2015;50(5):338-344. doi:10.1016/j.jcjo.2015.07.005

52. Vizzeri G, Weinreb RN. Cataract surgery and glaucoma. Curr Opin Ophthalmol. 2010;21(1):20-24. doi:10.1097/ICU.0b013e328332f562
Clinical Ophthalmology

\section{Publish your work in this journal}

Clinical Ophthalmology is an international, peer-reviewed journal covering all subspecialties within ophthalmology. Key topics include: Optometry; Visual science; Pharmacology and drug therapy in eye diseases; Basic Sciences; Primary and Secondary eye care; Patient Safety and Quality of Care Improvements. This journal is indexed on PubMed
Dovepress

Central and CAS, and is the official journal of The Society of Clinical Ophthalmology (SCO). The manuscript management system is completely online and includes a very quick and fair peer-review system, which is all easy to use. Visit http://www.dovepress.com/ testimonials.php to read real quotes from published authors. 